Elaine Marie A. Lagura, MD

Eduardo C. Yap, MD

Anna Victoria G. Garcia, MD

Department of Otolaryngology Head and Neck Surgery Ospital ng Makati
Correspondence: Dr. Eduardo C. Yap

Department of Otolaryngology Head and Neck Surgery

5th Floor, Ospital ng Makati

Sampaguita Street, Brgy. Pembo, Makati City 1208

Philippines

Phone: (632) 8826316 local 309

Email: osmakenthns@yahoo.com

Reprints will not be available from the authors.

The authors declared that this represents original material that is not being considered for publication or has not been published or accepted for publication elsewhere, in full or in part, in print or electronic media; that the manuscript has been read and approved by all the authors, that the requirements for authorship have been met by each author, and that each author believes that the manuscript represents honest work.

Disclosures: The authors signed disclosures that there are no financial or other (including personal) relationships, intellectual passion, political or religious beliefs, and institutional affiliations that might lead to a conflict of interest.

\section{Augmentation Rhinoplasty with Rib Cartilage Graft}

\author{
ABSTRACT \\ Objective: To investigate the outcome and complications of augmentation rhinoplasty with rib \\ cartilage grafts. \\ Methods: \\ Design: Retrospective study \\ Setting: Tertiary Government Hospital \\ Subjects: Patients who underwent dorsal nasal augmentation with autologous rib \\ cartilage grafts between June 2008 and October 2012.
}

Results: A total of 12 patients ( 3 male, 9 female) were included in the study. Mean age was 29 years. Seven were cases of primary simple rhinoplasty with four cases of revision (previously using alloplastic materials) and one case of trauma. Indications for the procedure were all cosmetic. There was no incidence of infection, both in the donor and recipient sites, warping of the graft, graft extrusion, resorption, pneumothorax, chest wall deformity or prolonged edema. Postoperative pain in the donor site was relieved by oral pain medications. No revision surgery was required.

Conclusion: Costal cartilage is a good option for structural support of the nose. In our experience patients have become wary of the complication of allografts and have opted to use autografts. The surgeon's knowledge of the nasal anatomy as well as his or her experience with autologous grafts plays a major role in avoiding post-operative morbidity.

Keywords: Rhinoplasty, rib graft, costal cartilage graft, Southeast Asian nose

The nose occupies a central position in the face. Any deformity of the nose can lead to functional and psychological disability. ${ }^{1}$ Rhinoplasty aims to achieve nasal balance and establish harmony with the face while preserving a functional nasal airway. Graft and implant materials are used primarily to maintain or strengthen the structural framework to provide contour or camouflage defects and to restore the nose to an aesthetic ideal. ${ }^{2}$

There are three broad categories of graft and implant materials currently available for rhinoplasty: autografts from the patient's own tissues; homografts from tissues obtained from a different donor of the same species; and alloplasts. ${ }^{2}$ Autogenous cartilage has generally been 


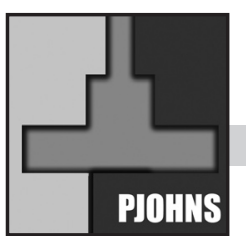

considered the gold standard grafting material in reconstructive septorhinoplasty for volume filling and structural support. It can be harvested from the nasal septum, the auricle or the rib but costal cartilage is considered the best graft material in patients requiring major reconstruction. ${ }^{3}$ Costal cartilage provides the advantages of a large volume of graft material with excellent structural support and low rate of complications such as resorption, infection and extrusion compared to homografts and alloplastic implants. The disadvantages observed include warping and potential donor site morbidities including pneumothorax, scar visibility and chest wall deformity.-3

\section{METHODS}

A retrospective analysis was done on patients who underwent augmentation rhinoplasty with rib cartilage graft in a tertiary government hospital from June 2008 to October 2012. The patients were admitted with detailed history, clinical examination, routine investigations and special investigations including photography. Consent was obtained from each patient for surgical demonstration as well as the possible public viewing of videos and pictures taken during the procedure. (Appendix A) Additional consent was obtained for the publication of pictures for this study.

All procedures were carried out under general anesthesia and were covered by prophylactic intravenous antibiotics. The open rhinoplasty was performed by the same senior surgeon on all patients.

\section{Rib cartilage harvest}

In male patients, the incision was made over the seventh costal cartilage and in women under the breast crease to hide the scar. Although either side may be utilized, all of our patients had their grafts harvested from the right side. After the skin incision, the overlying muscles were spread and retracted until the underlying costal cartilage

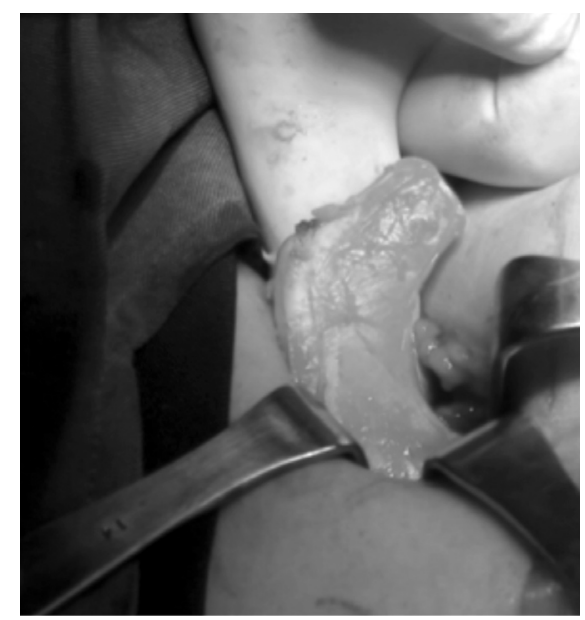

Figure 1. Harvest of $7^{\text {th }}$ intercostal rib

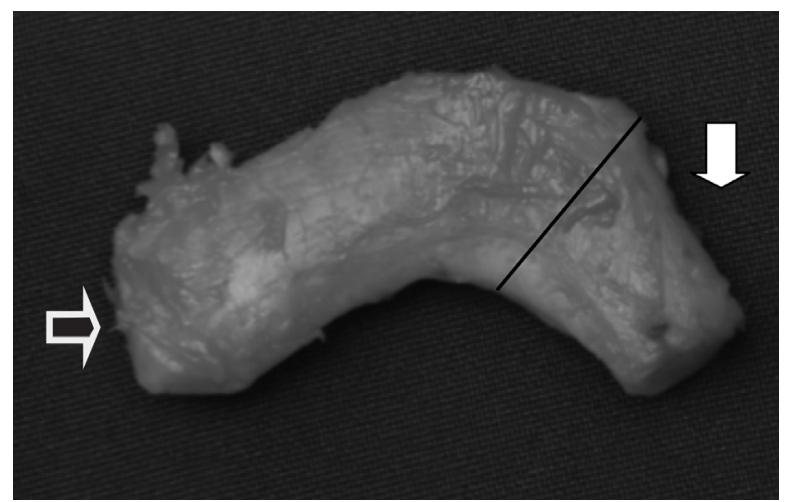

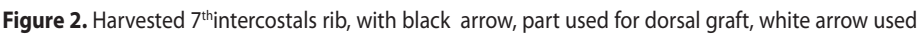
for other enhancement grafts.

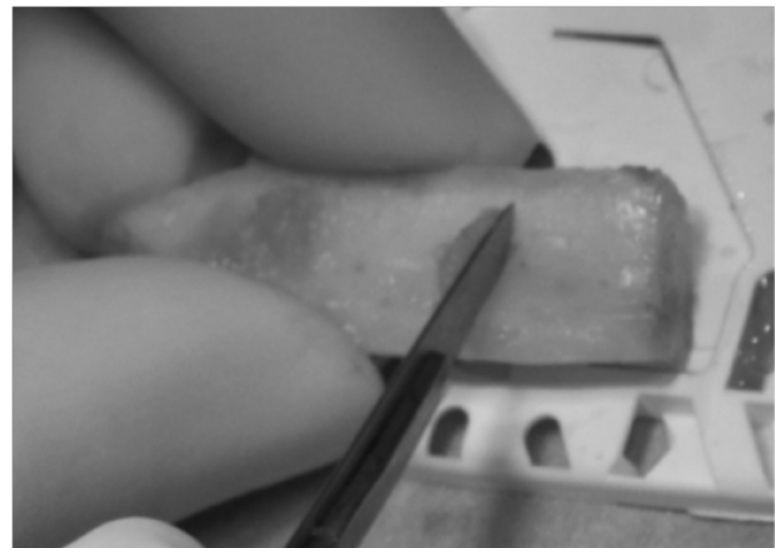

Figure 3. Shaping of the dorsal graft

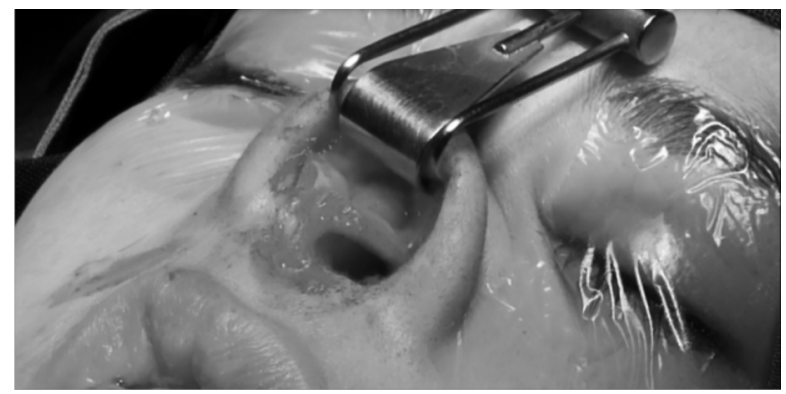

Figure 4. Exposure using open technique

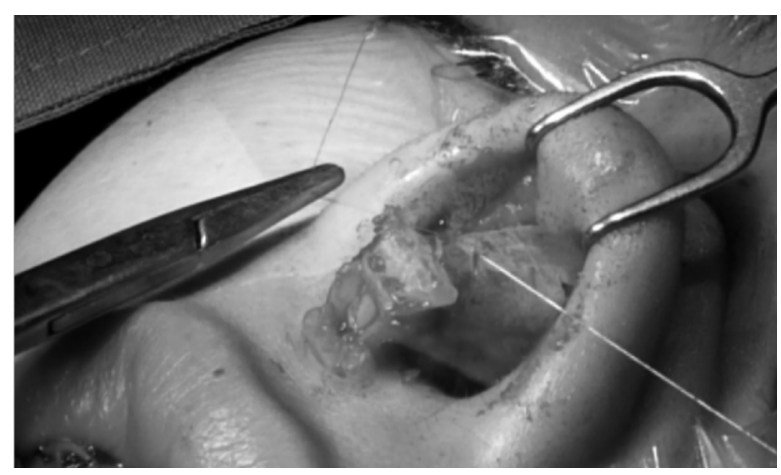

Figure 5. positioning of rib graft 
ORIGINAL ARTICLES

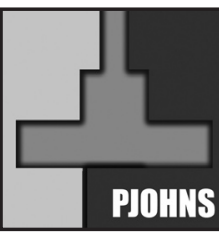

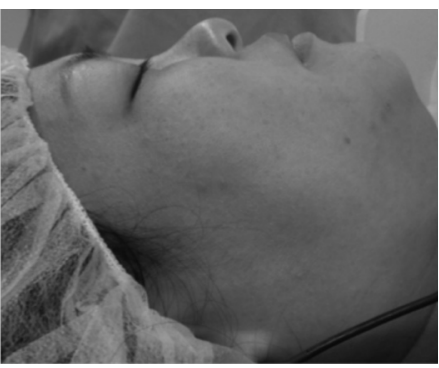

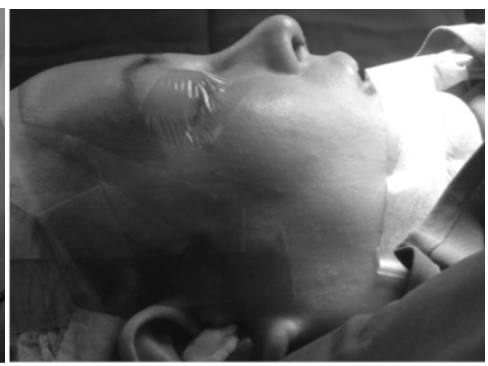

Figure 6. Immediate pre operative and post operative

was exposed. The perichondrium was cut and subperichondrial dissection was done using a periosteal elevator. The cartilaginous rib was separated from its medial attachment near the sternum and laterally from the bony rib. A partial-thickness incision was made perpendicular to the long axis of the rib. The cartilaginous incision was then completed with the sharp end of a Freer elevator. (Figure 1) Once the cartilage segment was released both medially and laterally, the graft was easily removed from the wound and placed in sterile saline with gentamicin until the surgeon was ready for shaping. Water leak test was performed to make sure there was no tear in the pleura. The longer end of the cartilage was used for dorsal augmentation while the shorter segment could be utilized for support or contour grafts. (Figure 2) In shaping of the dorsal graft, cross-hatching of the cartilage was done to prevent warping. (Figure 3)

\section{Rhinoplasty}

An open approach was utilized. (Figure 4) The columellar flap was freed with scissors dissecting up to expose the dome of the lower lateral cartilage. Soft tissue dissection was continued in the superficial muscular aponeurotic system plane and in the subperiostal plane over the bony vault for adequate access to the nasal dorsum. (Figure 5) Septoplasty was done in cases where there was septal deviation leaving a $10 \mathrm{~mm} \mathrm{~L}$-strut. The shorter limb of the harvested costal cartilage was fashioned into $2 \mathrm{~mm}$ sheets for use as septal extension graft, spreader graft and tip grafts. Depending on the vector of the new tip, the septal extension graft was sutured to the caudal end of the septum using PDS 5-0. The lower lateral cartilage was fixed to the caudal end of the septal extension graft. Further enhancement of the tip was done using various tip grafts e.g. Shield and onlay graft. (Figure 6) Alar lift procedure via sail excision and alar base plasty were done when needed.

All of the patients were discharged one day post operatively with continuation of antibiotics for one week and adequate analgesics. Follow-up was done 5-7 days post operatively for the removal of splint and sutures, then again at one month, 3 months, six months, 12 months and yearly thereafter with photo documentation. (Figures 7,8 )
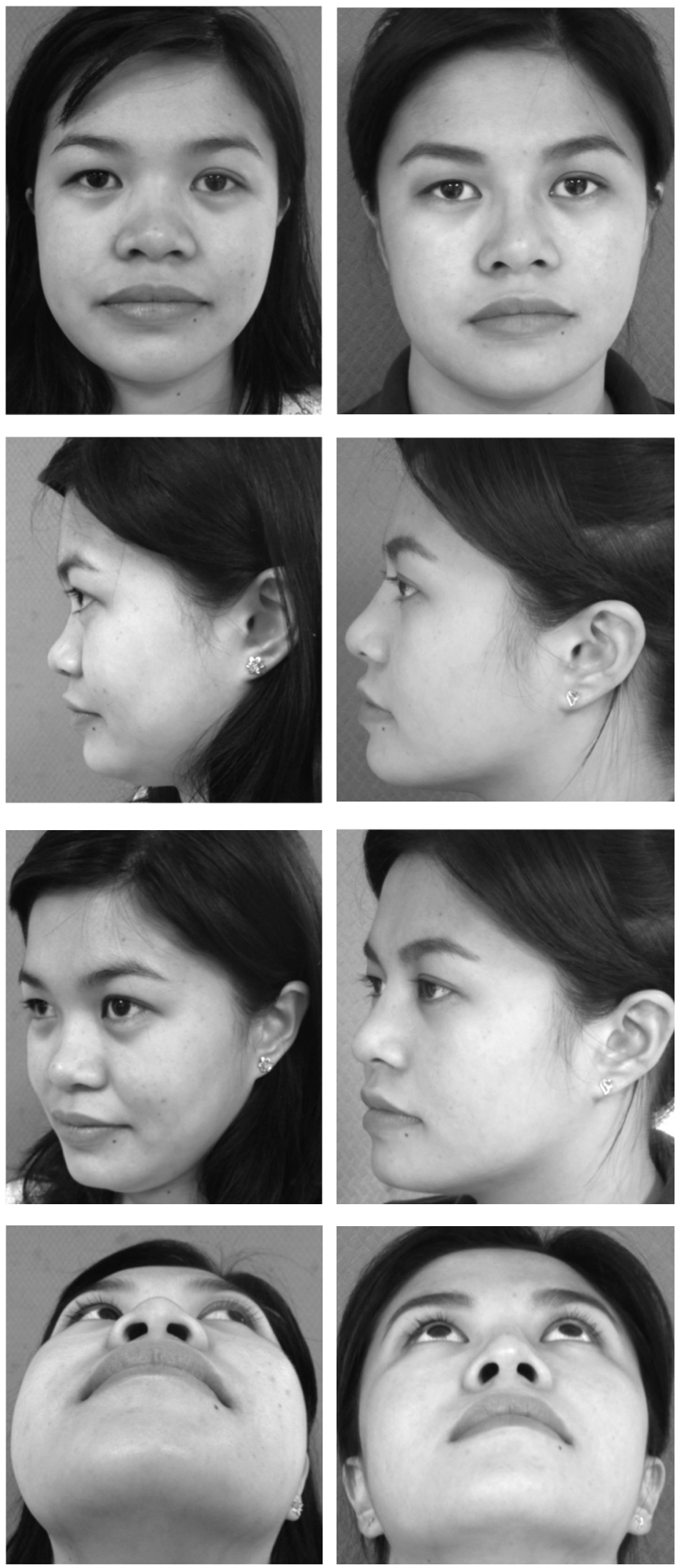

Figure 7. Pre-operative and 6 months post operative picture. Photos printed in full with permission. 


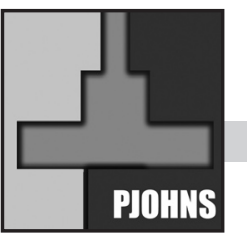

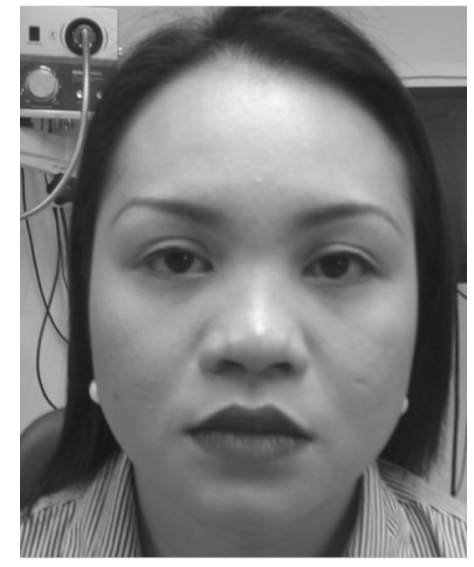
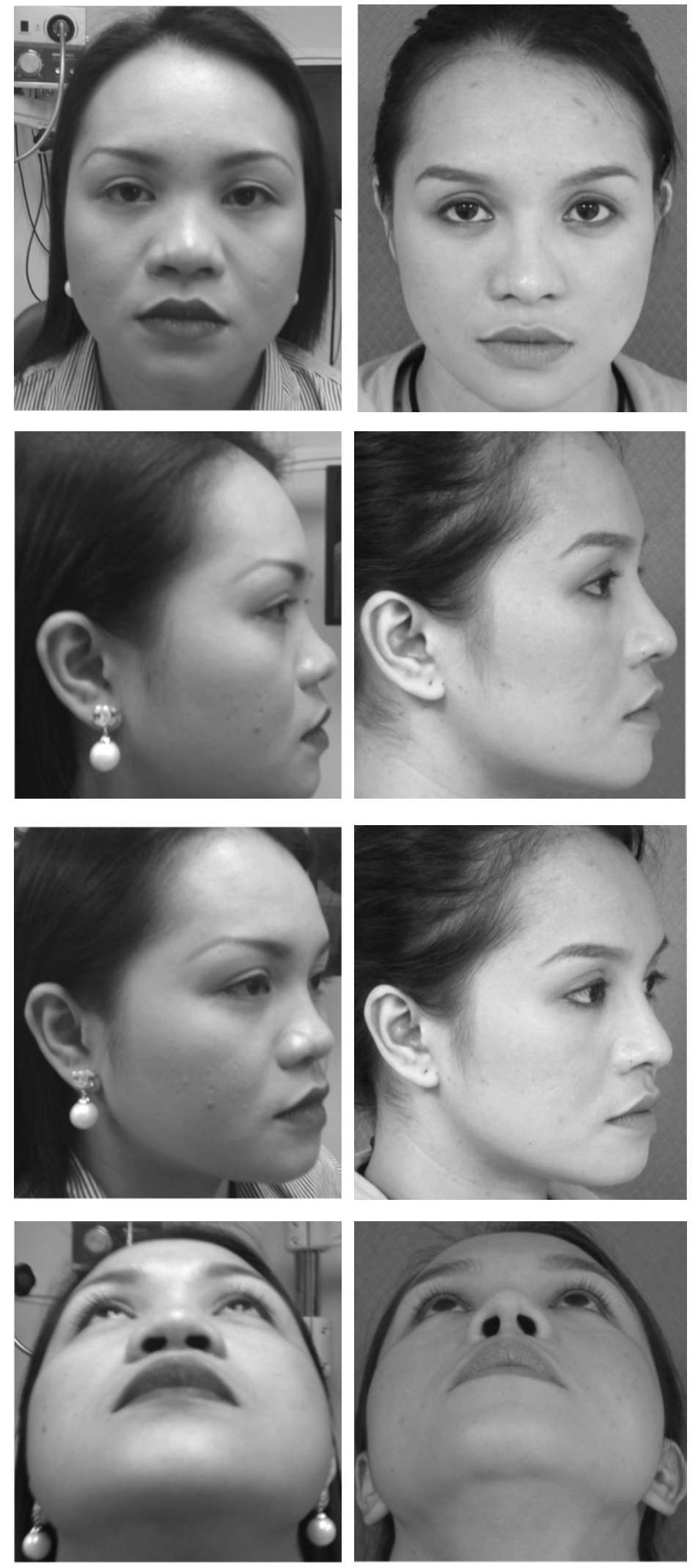

Figure 8. Pre operative and 2 years post operative picture. Photos printed in full with permission

\section{RESULTS}

A total of 12 patients ( 3 male and 9 female) were included in the study. Age ranged from 23 to 54 years old with a mean age of 29 years. There were four cases of revision rhinoplasty previously using alloplastic materials and one case of trauma. The rest of the cases were primary simple rhinoplasty. Indications for the procedure were all cosmetic. There was no incidence of infection, both in the donor and recipient sites, warping of the graft, graft extrusion, resorption, pneumothorax, chest wall deformity or prolonged edema. Patients complained of minimal post-operative pain in the donor site relieved by oral pain medication. Patients usually noted the pain to be maximal at 3 to 5 days and tolerable at 7 to 10 days. No revision surgery was required.

\section{DISCUSSION}

The goal of septorhinoplasty is reconstruction of the nasal skeleton to provide adequate structural support allowing optimum functioning of the nasal airway while achieving an aesthetically pleasing result with the rest of the face. To obtain aesthetically pleasing results, ensure patient satisfaction and minimize complications, the rhinoplasty surgeon must possess a thorough knowledge of nasal anatomy and ideal facial aesthetic proportions. The surgeon must be familiar with all types of graft material and the current methods to correct nasal deformities. ${ }^{1,4}$ Understanding the use of autologous, homologous and alloplastic materials for grafting and implantation purposes has become a necessity in the armamentarium of the rhinoplasty surgeon. ${ }^{2}$

Graft and implant materials are used primarily to maintain or strengthen the structural framework to provide contour or camouflage defects and to restore the nose to an aesthetic ideal. The ideal graft or implant material is biocompatible and possesses physical properties and long-term stability devoid of complications. Cartilage is nearly the ideal implantation material by its excellent biotolerance having low infection and extrusion rates. Cartilage possesses excellent elasticity, resistance, is easy to shape, has good vitality even with poor blood supply and a minimal resorption rate.

The rib offers an abundant supply of cartilage for use in virtually every aspect of rhinoplasty and is the preferred donor site when rigid support is necessary. The most significant advantage of rib cartilage is that grafts can be produced with considerable versatility with respect to shape, length and width. This facilitates reconstruction of the nasal framework in patients with virtually all types of functional and aesthetic requirements. ${ }^{5,6}$

Uppal et al. did a retrospective study on 42 patients who underwent coastal cartilage harvest for ear reconstruction. They noted that donor site pain and clicking sound were the most common complaints. Donor site scarring and deformity were acceptable for most patients but five 
underwent reconstruction to correct the deformity.

In a study by Cakmak et al. 20 patients with severe nasal deformity, 19 of which were revision cases received autogenous costal cartilage grafts. They had one patient with early wound infection and three cases of minor warping. They did not observe graft extrusion or resorption. Other than temporary pain, there were no donor site morbidities encountered.

Saeed likewise did a retrospective study of 60 patients who underwent augmentation rhinoplasty with rib cartilage harvest for patients with saddle nose deformity with no incidence of graft resorption, infection and extrusion as with our study. They had one patient with pneumothorax (2\%), another with marginal show (2\%) and five patients with keloid formation (8\%).

Another study by Park et al. noted a complication rate of $12 \%$ : of the 83 patients included in the study, five developed post-operative infection controlled by intravenous antibiotics and five had a warped graft.

In our study, there were no incidence of post-operative infection in both donor and recipient sites, warping of the graft, graft resorption, graft extrusion, pneumothorax, chest wall deformity or prolonged edema. The only donor site morbidity we encountered was pain that was adequately managed with oral analgesics.

We conclude that costal cartilage is a good option for structural support of the nose especially in patients with previous allografts. In our experience patients have become wary of the complications of allografts and have opted to use autografts. The surgeon's knowledge of nasal anatomy as well as his or her experience with autologous grafts plays a major role in avoiding post-operative morbidity.

\section{REFERENCES}

1. Tahir M, Abir S, Ullah F. Rib Graft Rhinoplasty for the depressed dorsum of the nose. JPMI. 2006; 20(3):264-267.

2. Lin G, Lawson W. Complications using grafts and implants in rhinoplasty, Operative Techniques in Otolaryngology. 2007; 18: 315-323

3. Morrieti A, Scuito S. Rib grafts in septorhinoplasty. Acta Otorhinolaryngol Ital. 2013 Jun;33(3):190195.

4. Saeed M. Costal cartilage graft in augmentation rhinoplasty. APMC.2012 Jul-Dec; 6(2): 166170.

5. Gunter JP, Cochran CS, Marin VP. Dorsal augmentation with autogenous rib cartilage. Semin Plast Surg. 2008 May; 22( 2): 74-89.

6. Marin VP, Landecker A, Gunter JP. Harvesting rib cartilage grafts for secondary rhinoplasty. PlastReconstrSurg. 2008 Apr; 121(4): 1442-8.

7. Baladiang DEA, Olveda MB, Yap EC. The "sail" excision technique: A modified alar lift procedure for Southeast Asian noses. Philipp J Otolaryngol Head Neck Surg. 2010 Jan-Jun; 25( 1): 31-37.

8. Lee M, Inman J, Ducic Y. Central segment harvest of costal cartilage in rhinoplasty. Laryngoscope 2011 Oct; 121(10):2155-2158

9. Al-Aziz A, AhmadH, Al-Leithy I. Autogenous Cartilage Grafts in Primary Rhinoplasty in the NonCaucasian Population , Egypt. J Plast Reconstr Surg. 2005 Jan; 29(1): 67-72.

10. Cakmak O, Ergin T. The versatile autogenous costal cartilage graft in septorhinoplasty. Arch Facial Plast Surg. 2002 Jul-Sep;4(3):172-6.

11. Uppal RS, Sabbagh W, Chana J, Gault DT. Donor-site morbidity after autologous costal cartilage harvest in ear reconstruction and approaches to reducing donor-site contour deformity. Plast Reconstr Surg. 2008 Jun; 121(6):1949-55.

12. Park JH, Jin HR. Use of autologous costal cartilage in Asian rhinoplasty. Plast Reconstr Surg. 2012 Dec; 130(6):1338-48.

13. Yap EC. Improving the hanging ala. Facial Plast Surg. 2012; 28(02): 213-217. 